\title{
Mechanisms of Bacterial Pathogenesis in Equine Endometritis
}

\author{
M. M. Wittenbrink. L. Hölzle and Antje-Katrin Baumeister \\ Institut für Medizinische Mikrobiologie, Infektions- und Seuchenmedizin, Ludwig-Maximilians-Universität München
}

\begin{abstract}
Summary
Bacteria that are established as uterine pathogens in the mare are only partially characterized with respect to virulence factors (mechanism of pathogenesis) that may contribute to the development of endometritis. The study of these mechanisms will be useful in defining many aspects of the complex interactions that occur between bacterial pathogens and the equine endometrium. Bacterial adherence is the primary prerequisite for infection. The role of the host cell cannot be ignored, since it obviously influences the regulation of microbial virulence factore e.g. adhesins as well as provides the microenvironment in which the bacteria can replicate and produce toxins that cause damage to the host. Detailed knowledge about host-pathogen interactions will lead to a better etiological assessment of uterine bacterial infections and probably lead to innovative strategies for therapy and prevention.
\end{abstract}

keywords: mare, endometritis, aetiology, pathogenesis, bacteria

\begin{abstract}
Pathogenitätsmechanismen bei bakteriellen Endometritiden der Stute
Die bei der Stute als uteropathogen bekannten Bakterien sind hinsichtlich der Virulenzfaktoren, die zur Endometritis füren (Pathogenesemechanismen) wenig charakterisiert. Die Untersuchung dieser Mechanismen trägt zum Verständnis der zahlreichen Aspekte komplexer Interaktionen zwischen pathogenen Bakterien und dem equinen Endometrium bei.

Voraussetzung für eine Infektion ist die Adhärenz der Bakterien. Eine weitere Rolle spieit die Wirtszelle, da sie sowohl die Regulation der mikrobiellen Virulenzfaktoren, z.B. Adhäsine, beeinflußt, als auch das Mikroenvironment schafft, in dem die für den Wirt schädliche Bakterienreplikation und Toxinproduktion vonstatten geht. Detaillierte Kenntnisse zur Wirt - Bakterien Interaktion führen zu einer verbesserten ätiologischen Klärung uteriner bakterieller Infektionen und können möglicherweise einen Beitrag liefern zur innovativen therapeutischen und präventiven Strategie.
\end{abstract}

Schlüsselwörter: Equine Endometritis, Ätologie, Pathogenese, Bakterien

\section{Introduction}

Equine endometritis schematically results from complex interactions between potential uterine bacterial pathogens and host factors. Host factors which may exert significant influences on the clinical outcome of a uterine infection include the genetic constitution and hormonal situation of the mare, the presence of anatomic deviations in the genital tract, age, and defense mechanisms such as phagocytosis and humoral immune mechanisms in uterine secretions. Deficencies in the local uterine immune system associated with increased uterine secretion or impaired mechanical removal of uterine fluid are suggested to be of major significance in the pathogenesis of equine endometritis. Those mares have only limited capabilities to eliminate bacteria gaining entrance to the uterus.

Bacteria involved in equine endometritis are classified as opportunistic pathogens because they often colonize the lower genital tract as well as a variety of extragenital locations in healthy horses but are normally kept from ascending transcervically into the uterus by host defences. Bacterial transfer to the uterus occurs accidentally during mating or artificially due to transcervical gynecological manipulations. The opportunistic uterine pathogens comprise a small panel of maximum 10 different bacterial species with Strepto- coccus (Sc.) equi subspecies (ssp.) zooepidemicus being the most significant. The etilogic role of these bacteria is mainly based on their consistent recovery from the uterus with inflammatory alterations. Members of the indigenous equine genital microflora, which comprises among others at least 19 species of gramnegative non-fermenting bacteria and 12 species of grampositive irregular nonsporing rods, are easily introduced into the uterus e.g. during mating, but give only rarely rise to an endometrial infection. Thus, bacteria, which are capable to establish endometritis are considered to differ from the physiological mucosa-colonizing genital microflora by several traits (products or strategies) that promote colonizing and causing damage to the equine endometrium. The concept that a few virulence mechanisms are sufficient to explain why a bacterium can cause endometritis in the mare may oversimplify the complex nature of this disease. Nevertheless, it is still necessary to elucidate those bacterial features in detail e.g. to develop new therapeutic or vaccination strategies. According to Salyers and Whitt (1994), bacterial virulence factors can be loosely classified into two categories: those that promote bacterial colonization and invasion of the host and those that cause damage to host tissues. In the following, viru- 
lence factors of bacteria well known as major uterine pathogens in the mare are reviewed.

\section{Bacterial adherence}

Bacterial adherence to host cells is the primary prerequisite in the development of infection. Virtually all known bacterial pathogens have the ability of attaching themselves firmly to the surface of host cells. This attachment is the result of interactions between structures on the bacterial surface (adhesins) and receptors on the host cell. Bacteria use several strategies for adherence, the most investigated adherence mechanism being the binding of bacterial surface appendages such as pili (fimbriae) to host cell receptors. Pili are long, flexible, rod-shaped protein structures that extend outward from the gramnegative bacterial surface. Binding of the pilus tip to receptor structures on the host cell surface may be mediated either by the main pilus protein constituent designated as pilin or by specialized tip structures distinct from pilin. Recent models imply that pili mediate an initial loose contact between bacterial and host cell leading to a more tightly binding mediated by other bacterial surface proteins. The significance of pili in bacterial adhesion is supported by studies showing that nonpiliated mutants of Pseudomonas (Ps.) aeruginosa exhibit decreased virulence in animal models (Hazlett et al., 1991). Moreover, purified pili and antibodies against pili are shown to block bacterial adhesion to epithelial cells (Rudner et al., 1993). The assembly of pili occurs in many gramnegative bacterial pathogens e.g. Escherichia (E.) coli or Klebsiella (K.) pneumoniae which are often recovered as etiological agents from equine endometritis. Recent in vitro studies have shown that Taylorella $(T$.) equigenitalis, the etiological agent of CEM, quickly adheres to epithelial cells of equine origin. The hypothesis that $T$. equigenitalis requires rapid adherence to the endometrial mucosa to avoid beeing flushed away is further confirmed by in vivo studies which have demonstrated that adherence of this bacterium to cilia of epithelial cells occurs by means of pili, although pili have not yet been observed in vitro (Bleumink-Pluym et al., 1996; Kanemaru et al., 1992). Alternatively, bacteria can make nonfimbrial adhesins. These adhesins do not have the ordered structure characteristics of pili and are, therefore, designated as afimbrial adhesins. The cell wall of Sc. pyogenes, a group A streptococcus pathogenic for humans, carries a non-pilus adhesin that mediates the binding of fibronectin, a protein found on the surface of many mammalian cells. Group $C$ streptococ$\mathrm{ci}$ including Sc. equi spp. zooepidemicus, the most frequent cause of endometritis in mares, have also been shown to bind to fibronectin. Fibronectin-binding presumedly plays a role in bacterial adherence to the mucosa but it still remains unknown whether and to which extend adhesive ligands contribute to the pathogenic interaction of Sc. equi ssp. zooepidemicus with the equine endometrium.

Most studies of bacterial adherence to mammalian host cells have focused on the binding by bacterial adhesive ligands that interact with host cell receptors. Bacteria can also form dense, matrix-embedded multiorganism layers on surfaces (biofilms). Biofilms are important for the survival of free-living bacteria e.g. in aqueous environments, but there is only little evidence concerning the possible involvement of this type of bacterial adherence in animal disease. By means of electron microscopy, biofilms have been detected in the mouth, in the intestine and in the vagina. The indigenous microflora of these sites may be organized as biofilms to prevent pathogens from gaining access to the mucosal surface. Dental plaque is an example of a biofilm known to cause disease. In the uterus bacteria are suggested to form biofilms in the presence of an intrauterine substrate probably uterine secretions (Gard et al., 1993). Despite the presence of immunoglobulins, complement and phagocytic cells, bacteria can accumulate in this matrix and cause among others toxin-mediated membrane damages of a prolonged nature in the endometrial epithelium. Bacteria organized in biofilms are partially protected from phagocytosis and are more resistant to antiseptics and antibiotics than bacteria forming a homogeneous suspension.

\section{Bacterial mechanisms for evading host defenses}

Pathogenic bacteria are capable of extracellular survival in vivo by evasion complement activation, host's antibody response, and phagocytosis. In certain bacterial pathogens, evasion of host defenses is mediated by capsules. Capsules are unstructured accumulations of polymers, mostly polysaccharides, that form the outermost layer of certain gramnegative as well as grampositive bacteria. The growth of encapuslated bacteria triggers the production of antibodies against capsular antigens, leading to opsonization and phagocytosis. However, certain bacteria can avoid this effective host response having capsules from polysaccharides that are also normal constituents of host tissues like sialic acid or hyaluronic acid. These kind of capsules are non-antigenic thus preventing the production of specific antibodies by the host. Hyaluronic acid capsules are found in equine group $\mathrm{C}$ streptococci, namely the main uterine pathogen Sc. equi ssp. zooepidemicus. It appears that in Sc. equi ssp. zooepidemicus the expression of factors mediating evasion from host defenses probably undergoes phase variation. A higher proportion of streptococcal isolates from the equine cervix exhibit spontaneous phase variation in vitro among at least two clearly discernible phenotypes (presumedly encapsulated and non-capsulated).

$\mathrm{S}$. equi ssp. equi, the cause of strangles in the horse, produces a fibrillar surface protein called $M$ protein analogous to the $M$ proteins of group A streptococci. M proteins are anchored in the cell wall and extend into and through the capsule. The $M$ protein is a major virulence factor and also a protective antigen. It has antiphagocytic properties by mediating degradation of $\mathrm{C} 3 \mathrm{~b}$ and by directly binding fibrinogen (Timoney et al., 1995). In Sc. equi ssp. zooepidemicus M-like proteins are found which stimulate specific opsonizing antibodies and protect mice against challenge with homologous strains. Virtually all isolates of Sc. equi ssp. zooepidemicus from the equine genital tract reveal the presence of the gene encoding $\mathrm{M}$-like proteins but it reamains unclear whether this 
protein is expressed by all strains. However, in group A streptococci mutants lacking $M$ protein are less virulent because more susceptible to phagocytosis than the wild type. Thus, $M$ proteins and $M$-like proteins are suggested to be indispensible to undergo phagocytosis successfully. RFLP analysis of a sample of 50 strains of Sc. equi ssp. zooepidemicus revealed considerable heterogeneity of the encoding gene suggesting that high antigenic diversity and probably antigenic variation of $\mathrm{M}$-like proteins may help those bacteria to resist clearance by the host immune response.

Some bacteria are characterized by an ability to invade nonphagocytic cells as an early step in pathogenesis. Other bacteria have evolved mechanisms to survive within phagocytic cells. The molecular mechanisms involved in these processes are currently under intensive study. Staphylococcus (S.) aureus, addmittedly a minor pathogen in equine endometritis, is able to survive and multiply within phagocytic cells thus escaping clearance by host immunologic defenses and also by antibiotics (Maurin and Raoult, 1993). T. equigenitalis, the cause of CEM, is able to invade and replicate in an epithelial cell line established from equine dermis (Bleumink-Pluym et al., 1996). The invasiveness of T. equigenitalis strains tested in the cell culture model seemed to be associated with the contagiousness of the infection, whereas the replication index of intracellular $T$. equigenitalis was obviously correlated with severity of the clinical symptoms of CEM. However, evidence that invasiveness and intracellular persistence of bacteria is of any significance in the outcome of chronic endometritis is still missing.

\section{Bacterial toxins}

Many bacteria produce proteins which are excreted into the medium and can exert toxic effects to mammalian cells. These proteins are designated as exotoxins, a term meant to differentiate the excreted toxic substances from the socalled endotoxin, a constituent of the lipopolysaccharide (LPS) of gramnegative bacteria. Inflammation of the endometrium can be induced by receptor-mediated adhesion of gramnegative bacteria in synergy with LPS. After attachment, the lipid A moiety of bacterial LPS triggers the production of acute-phase reactants and chemoattractants with the consequence of increased recruitment to the mucosa of polymorphonuclear leukocytes. Moreover, certain gramnegative and grampositive bacteria that are recovered from equine endometritis elaborate a range of exotoxins that might cumulatively damage the endometrium. However, bacteria recovered from equine endometritis have not systematically been examined for toxigenic properties and thus only little is known about the significance of bacterial exotoxins in the pathogenesis of equine endometritis. Sc. equi ssp. zooepidemicus and St. aureus elaborate exotoxins that exhibit hemolytic activity on mammalian erythrocytes in vitro and are therefore readily detected by bacteriologic culture. The staphylococcal $\alpha$-hemolysin is a pore-forming toxin that assembles on the surface of a broad range of eukaryotic cells and creates an aqueous transmembrane pore. Primary mechanisms of cell death are permeabilization of cell membranes to ions, water, and low molecular weight molecules, and cell lysis (Song et al., 1996). St. aureus isolates from equine metritis belong to biotype B and C (Shimizu et al., 1991). Most of biotype B strains produce $\alpha$-hemolysin whereas biotype $C$ strains are variable, but all biotype $C$ strains produce $\beta$-hemolysin that exhibits damage to eukaryotic cells by phospholipase $\mathrm{C}$ activity. Relatively little is known in detail about the structure and function of the $\beta$-hemolysin of Sc. equi ssp. zooepidemicus. The $\beta$-hemolysin of group $\mathrm{C}$ streptococci shows lower hemolytic activity than streptolysin $\mathrm{S}$, but did not differ from streptolysin $S$ with respect to the binding and release of hemoglobin (Suzuki et al., 1995). Thus it may be concluded that the $\beta$-hemolysin of Sc. equi ssp. zooepidemicus also exerts membrane-damaging activities on cells others erythrocytes. However, as is the case for the streptococcal $\beta$-hemolysin, the role of most of the bacterial exotoxins in the development of endometritis remains to be elucidated.

\section{References}

Bleumink-Pluym, N.M. E.A. Ter Laak, D.J. Houwers, and B.A.M. Van der Zeijst (1996): Differences between Tayloreila equigenitalis strains in their invasion of and replication in cultured cells. Clin. Diagn. Lab. Immunol. 3, 47-50

Gard, P.R., J.S. Malhi, and G.F. Hanlon (1993): Uterine contamination in the guinea pig following transcervical uterine monofilament inser tion. Gynecol. Obstet. Invest. 35, 49-52

Hazlett, L.D., M.M. Moon, A. Singh, R.S. Berk, and X.L. Rudner (1991): Analysis of adhesion, piliation, protease production and ocular infectivity of several Pseudomonas aeruginosa strains. Curr. Eye Res. 10, 351-362

Kanemaru, T., M. Kamada, T. Anzai. T. Kumanomido, H. Yoshikawa, and T. Yoshikawa (1992): Electronmicroscopic observations of $T$. equigenitalis with pili in vivo. J. Vet. Med. Sci. 54, 345-347

Maurin, M. and D. Raoult (1994): Phagolysosomal alkalinazation and intracellular killing of Staphylococcus aureus by amikacin. J. infect. Dis. $169,330-336$

Rudner, X.L., R.S. Berk, and L.D. Hazlett (1993): Immunization with homologous Pseudomonas aeruginosa pili protects against ocular disease. Reg. Immunol. 5, 245-252

Salyers, A.A and D.D. Whitt (1994): Bacterial pathogenesis - a molecular approach. ASM Press, Washington, DC 2005

Shimizu, A., J. Kawano, J. Ozaki, N. Sasaki, S. Kimura, M. Kamada, S. Anzai, H. Saito, and H. Sato (1991): Characteristics of Staphylococcus aureus isolated from lesions of horses. J. Vet. Med. Sci 53,601-606

Song, L., M.R. Hobaugh, C. Shustak, S. Cheley, H. Bayley, and J.E. Gouaux (1996): Structure of staphylococcal (-hemolysin, a heptameric transmembrane pore. Science 274, 1859-1866

Suzuki, J., E. Yoshihara, S. Kobayashi, M. Fukuyama, S. Kisikawa, M. Hara, and K. Tabuchi (1995): Properties of a hemolysin produced by group $C$ streptococci isolated from hemolytic streptococcal infection in formosan squirreis. Kansenshogaku Zasshi 69, 327-332

Timoney, J.F., Walker, J., M. Zhou, and J. Ding (1995): Cloning and sequence analysis of a protective $M$-like protein gene from Streptococcus equi subsp. zooepidemicus. Infect. Immun. 63, 1440-1445

\section{Prof. Dr. M.M. Wittenbrink}

Institut für Medizinische Mikrobiologie

Infektions- und Seuchenmedizin

Ludwig-Maximilians-Universität München

Veterinästr. 13

80539 München

Tel: 089-21802596 